Article

\title{
Application of Optimized Adaptive Chirp Mode Decomposition Method in Chirp Signal
}

\author{
Junyuan Wang ${ }^{1,2}$, Huihui He ${ }^{1,2}$, Zhijian Wang ${ }^{1,2,3, *}$, Wenhua Du ${ }^{1,2}{ }^{-}$, Nengquan Duan ${ }^{1,2}$ \\ and Ziying Zhang ${ }^{4,5}$ \\ 1 School of Mechanical Engineering, North University of China, Taiyuan 030051, China; \\ wangyi01161013@163.com (J.W.); hehuihui124@163.com (H.H.); dwh@nuc.edu.cn (W.D.); \\ jianxuanyuan2012@163.com (N.D.) \\ 2 Key Laboratory of Advanced Manufacturing Technology of Shanxi Province, Taiyuan 030051, China \\ 3 School of Mechanical Engineering, Xi'an Jiaotong University, Xi'an 030619, China \\ 4 School of Mechanical Electronic \& Information Engineering, China University of Mining and Technology, \\ Beijing 100083, China; tbp1600401010@student.cumtb.edu.cn \\ 5 Shanxi Institute of Energy, Taiyuan 030006, China \\ * Correspondence: wangzhijian1013@163.com
}

Received: 11 April 2020; Accepted: 21 May 2020; Published: 27 May 2020

\begin{abstract}
The adaptive chirp mode decomposition method has a good effect on processing chirp signals. The parameter $\alpha$ controls the smoothness of the output signal. Too small an $\alpha$ will cause a smooth output signal. The parameter $\beta$ controls the instantaneous frequency (IF). If too small a $\beta$ value is used, the output IF will be very smooth. However, rapidly changing IFs require a relatively large $\beta$. However, the choice of $\alpha, \beta$ is artificially set, and there are errors in practical applications. Therefore, it employs the state transition algorithm to adaptively optimize $\alpha, \beta$ to improve the signal-to-noise ratio (SNR) and resolution of the signal. First, as the species number of the state transition algorithm method is set artificially and has a long running time, this paper proposes a Rastrigin optimization test equation to test the optimization time of different species and determine the number of optimal species; second, the state transition algorithm determined by the number of species is employed to adaptively find the $\alpha, \beta$ in the adaptive chirp mode decomposition algorithm; finally, the optimized adaptive chirp mode decomposition method is applied to the simulation signal and chirp signal from marine animals to verify the proposed method.
\end{abstract}

Keywords: ACMD; state transition algorithm; chirp signal; SNR

\section{Introduction}

In nature, a large number of nonlinear chirp (NC) signals are generated every day, such as human communication sounds, and the ultrasonic waves from bats, dolphins, and whales, etc. [1-4]. In the radar system [5,6], if the sinusoidal signal is transmitted and the target is in uniform-acceleration motion, the echo signal can be approximately regarded as the NC signal. In communication systems [7-9], some clutter and interference can be regarded as NC signals. As NC signals and their parameters have practical physical meaning in many fields, the research on NC signals detection and parameter estimation is of great significance.

Most decomposition methods extract each signal mode directly in the time domain, of which empirical mode decomposition (EMD) [10-13] is the most famous one. EMD is a completely data-driven method that can adaptively decompose complex NCs into simple modes. Although EMD is sensitive to noise and lacks mathematical models, it has been widely used in various fields. On the basis of EMD, the methods of EMD and ensemble empirical mode decomposition (EEMD) have been proposed; 
however, these approaches only address part of the problem, and some challenging issues remain to be addressed. For instance, these improved methods based on EMD have a poor resolution for split-off mode.

To further understand the characteristics of NC signals, it is necessary to extract each nonlinear chirp model from the signal data. In the past few decades, many methods have been developed to decompose NCs. These methods implement signal decomposition in the time, frequency, and time-frequency (TF) domains. From the TF plane, we can observe time-varying features and perform signal decomposition. However, due to the limitation of the Heisenberg uncertainty principle, the TF representation generated by conventional methods is usually fuzzy, and it is impossible to provide accurate TF representation for time-varying signals. The disadvantages of traditional TF methods severely limit their application in actual signal processing. In the past decades, TF has gradually developed many advanced methods, such as the reassignment method (RM) [14,15], synchrosqueezing transform (SST) [16,17], demodulated SST (DSST) [18,19], and high-order SST [20,21]. RM technology stimulates post-processing of traditional TF methods and is an effective way to obtain clearer TF results. However, the RM framework is based on a spectrogram, which means that RM results lose the ability to reconstruct the signal. The SST method not only enhances the TF resolution but also allows the signal to be reconstructed. On the contrary, when processing signals that change over time (such as a linear frequency-modulated signal or a non-linear frequency-modulated (FM) signal), SST cannot generate a concentrated TF result.

The time-frequency transform maps the chirp signal to the time-frequency domain. It is easy to observe the rule of frequency changes with time, which is conducive to signal detection and parameter estimation. The time-frequency transform can be used to describe the energy density of the signal at different times and frequency by constructing the joint function of time and frequency. At present, time-frequency transforms commonly used are the short-time Fourier transform (STFT) [22-25], Wigner-Ville distribution (WVD) [26,27], fractional Fourier transform [28,29], cubic phase function [30], and Zak transform (ZT) [31,32]. In addition, as the chirp signal usually presents an impact function of frequency linearly varying with time in the time and frequency domain, the linear detection and extraction method in image processing can be combined with the commonly used time-frequency transformation to estimate the initial frequency and frequency modulation slope. Chen [33] applied the VMD method to nonlinear chirp signals and achieved good results. However, as it processes the signals based on VMD, it is still limited to the determination of the number of signal decomposition layers. Chen [34] uses the matching tracking algorithm of the greedy algorithm to estimate each signal adaptively on the basis of Reference [33], which overcomes the defect of Variational nonlinear chirp mode decomposition (VNCMD). In reference [34], two important parameters $\alpha, \beta$ control the components of the output signal and instantaneous frequency (IF), respectively. However, as the selection of $\alpha$ and $\beta$ is artificially set, there are errors in practical applications.

Based on the above problems, this paper proposes to use the state transition algorithm (STA) [35] to adaptively determine $\alpha$ and $\beta$ to improve the resolution of the output signal. As the number of species in the STA is an artificial error, this paper proposes the Rastrigin optimization test equation to optimize the number of different species, and detects the time of each optimization (the number of species is $1-100$, in which the rotation operator, translation operator, expansion operator, and axesion operator are unchanged, and are set to $\alpha=1, \beta=1, \gamma=1, \delta=1$, respectively). In order to accurately determine the number of species, this paper takes the average value of 10,000 times the operation time of the same species optimization and compares the average optimization time of each species to determine the number of species corresponding to the minimum optimization time. Finally, the adaptive chirp mode decomposition (ACMD) method optimized in this paper is applied to the chirp signal from marine animals, which verifies the feasibility of the proposed method. 


\section{Basic Theory}

\section{1. $A C M D$}

ACMD was proposed by Chen on the basis of VNCMD, and they used a matching pursuit algorithm in the greedy algorithm to adaptively estimate each signal. The $\mathrm{i}$-th signal should be defined as follows:

$$
\begin{gathered}
\min _{a_{i}(t), b_{i}(t), \widetilde{f_{i}}(t)}\left\{\left\|a^{\prime \prime}{ }_{i}(t)\right\|_{2}^{2}+\left\|b^{\prime \prime}{ }_{i}(t)\right\|_{2}^{2}+\alpha\left\|s(t)-s_{i}(t)\right\|_{2}^{2}\right\} \\
\text { with } \mathrm{s}_{i}(t)=a_{i}(t) \cos \left(2 \pi \int_{0}^{t} \widetilde{f_{i}}(\tau) d \tau\right)+b_{i}(t) \sin \left(2 \pi \int_{0}^{t} \widetilde{f_{i}}(\tau) d \tau\right)
\end{gathered}
$$

where $\left\|s(t)-s_{i}(t)\right\|_{2}^{2}$ is the residual signal from which the current component signal is removed, and $\alpha>0$ is the weight of the coefficient. Like matching pursuit, ACMD greedily looks for signal components that extract the most energy from the input signal. Assuming that the signal is discrete in time $t=t_{0}, \cdots, t_{N-1}$ series, the discrete expression of Equation (1) is as follows:

$$
\min _{\mathbf{u}_{i}, \mathbf{f}_{i}}\left\{\left\|\Theta \mathbf{u}_{i}\right\|_{2}^{2}+\alpha\left\|\mathbf{s}-\mathbf{G}_{i} \mathbf{u}_{i}\right\|_{2}^{2}\right\}
$$

where $\boldsymbol{\Theta}=\left[\begin{array}{cc}\Omega & \\ & \Omega\end{array}\right]$ and $\Omega$ denotes the second-order difference matrix; $\mathbf{s}=\left[s_{i}\left(t_{0}\right), \cdots, s_{i}\left(t_{N-1}\right)\right]^{T}$, $\mathbf{a}_{i}=\left[a_{i}\left(t_{0}\right), \cdots, a_{i}\left(t_{N-1}\right)\right]^{T}, \mathbf{b}_{i}=\left[b_{i}\left(t_{0}\right), \cdots, b_{i}\left(t_{N-1}\right)\right]^{T}$, and $\mathbf{u}_{i}=\left[\mathbf{a}_{i}^{T}, \mathbf{b}_{i}^{T}\right]^{T}$.

$$
\begin{gathered}
\mathbf{G}_{i}=\left[\mathbf{G}_{i}, \mathbf{S}_{i}\right] \\
\mathbf{C}_{\mathbf{i}}=\operatorname{diag}\left[\cos \left(\varphi_{i}\left(t_{0}\right)\right), \cdots, \cos \left(\varphi_{i}\left(t_{N-1}\right)\right)\right] \\
\mathbf{S}_{\mathbf{i}}=\operatorname{diag}\left[\sin \left(\varphi_{i}\left(t_{0}\right)\right), \cdots, \sin \left(\varphi_{i}\left(t_{N-1}\right)\right)\right]
\end{gathered}
$$

where $\varphi_{i}(t)=2 \pi \int_{0}^{t} \widetilde{f_{i}}(\tau) d \tau$.

Equation (2) gives a matrix $\mathbf{G}_{i}$. The vector $\mathbf{u}_{i}$ can be estimated by solving the $l_{2}$ normalized least-squares problem. On the other hand, the phase function of the demodulation function contains information on the frequency function. The optimization problem of Equation (2) can be solved by an iterative algorithm, which can update the demodulated signal and frequency function in turn. For the $\mathbf{j}$-th iteration, the update of the vector $\mathbf{u}_{i}$ is as follows:

$$
\mathbf{u}_{i}^{j}=\left[\begin{array}{c}
\mathbf{a}_{i}^{j} \\
\mathbf{b}_{i}^{j}
\end{array}\right]=\underset{\mathbf{u}_{i}}{\operatorname{argmin}}\left\{\left\|\boldsymbol{\Theta} \mathbf{u}_{i}\right\|_{2}^{2}+\alpha\left\|\mathbf{s}-\mathbf{G}_{i}^{j} \mathbf{u}_{i}\right\|_{2}^{2}\right\}=\left(\frac{1}{\alpha} \boldsymbol{\Theta}^{T} \boldsymbol{\Theta}+\left(\mathbf{G}_{i}^{j}\right)^{T} \mathbf{G}_{i}^{j}\right)^{-1}\left(\mathbf{G}_{i}^{j}\right)^{T} \mathbf{s}
$$

The frequency function $\widetilde{f}_{i}^{j}(\tau)$ constructs $\mathbf{G}_{i}^{j}$, and the superscript.$^{j}$ indicates the number of iterations. In addition, the parameter $\alpha$ controls the smoothness of the output signal. At this time, the method is a TF band-pass filter, and the bandwidth of the filter is determined by $\alpha$. Too small an $\alpha$ will cause the filter to have a narrow bandwidth (i.e., a smooth output signal). The signal components can be estimated as follows:

$$
\mathbf{s}_{i}^{j}=\mathbf{G}_{i}^{j} \mathbf{u}_{i}^{j}
$$

Employing the demodulated signal obtained in Equation (6), the frequency increase can be estimated as follows:

$$
\Delta \widetilde{f_{i}^{j}}(t)=-\frac{1}{2 \pi} \frac{d}{d t}\left(\arctan \left(\frac{b_{i}^{j}(t)}{a_{i}^{j}(t)}\right)\right)=\frac{b_{i}^{j}(t) \times\left(a_{i}^{j}(t)\right)^{\prime}-a_{i}^{j}(t) \times\left(b_{i}^{j}(t)\right)^{\prime}}{2 \pi\left(\left(a_{i}^{j}(t)\right)^{2}+\left(b_{i}^{j}(t)\right)^{2}\right)}
$$


IF is a smoothing function and the growth of frequency should be smooth enough. Therefore, in practice, in order to reduce the influence of noise, a low-pass filter is used to process the signal growth. The IF may eventually be updated as follows:

$$
\mathbf{f}_{i}^{j+1}=\mathbf{f}_{i}^{j}+\left(\frac{1}{\beta} \mathbf{\Omega}^{T} \mathbf{\Omega}+\mathbf{I}\right)^{-1} \Delta \tilde{\mathbf{f}}_{i}^{j}
$$

where $\mathbf{f}_{i}^{j}=\left[\widetilde{f}_{i}^{j}\left(\tau_{0}\right), \cdots, \widetilde{f}_{i}^{j}\left(\tau_{N-1}\right)\right]^{T}, \Delta \tilde{\mathbf{f}}_{i}^{j}=\left[\Delta \widetilde{f}_{i}^{j}\left(\tau_{0}\right), \cdots, \Delta \widetilde{f}_{i}^{j}\left(\tau_{N-1}\right)\right]^{T}, \mathbf{I}$ is the identity matrix, $\mathbf{\Omega}$ is the second-order difference matrix, and $\left(\frac{1}{\beta} \Omega^{T} \Omega+\mathbf{I}\right)^{-1}$ denotes a low-pass filter. If too small a $\beta$ is used, the output IF will be very smooth. However, rapidly changing IFs require a relatively large $\beta$. Vector $\mathbf{G}_{i}^{j+1}$ can be updated by employing $\mathbf{f}_{i}^{j+1}$ (Equations (3)-(5)), and Equations (6)-(9) can be repeated in the next iteration.

As mentioned before, the parameters $\alpha, \beta$ control the components of the output signal and IF, respectively. In ACMD, in order to process the vibration signal with fast fluctuation IFs, it is recommended to use a relatively large $\alpha$ and $\beta\left(\alpha=1 \times 10^{-3}, \beta=1 \times 10^{-4}\right)$, and the termination threshold $v$ is set to $1 \times 10^{-8}$ according to the required accuracy. However, the choice of $\alpha, \beta$ is artificially set, and there are errors in practical applications. Therefore, this paper employs the state transition algorithm (STA) to adaptively optimize $\alpha, \beta$ to improve the signal-to-noise ratio (SNR) and resolution of the signal.

\subsection{State Transition Algorithm}

The STA [35] can optimize several parameters at the same time, and it is an efficient random optimization algorithm. This algorithm treats the result of the optimization problem as a state, and regards the process of searching in the search space as an evolutionary algorithm of the process of state transition. This algorithm uses four state operators of rotation, translation, expansion, and axesion to solve the continuous optimization problem. The algorithm has strong global search ability, fast convergence speed, and high optimization precision.

The STA can be written as:

$$
\left\{\begin{array}{l}
x_{k+1}=A_{k} x_{k}+B_{k} u_{k} \\
y_{k+1}=f\left(x_{k+1}\right)
\end{array}\right.
$$

where $x_{k} \in R^{n}$ and $x_{k+1}$ represent the current state and the transition state, respectively. $A_{k}$ and $B_{k}$ are state transition matrices and can be considered as the operator of the optimization algorithm. $u_{k}$ is a function related to the current state $x_{k}$ and the historical state. $f()$ denotes the objective function.

To solve the optimization problem of Equation (10), the four core operation operators are as follows:

Rotation operator:

$$
x_{k+1}=x_{k}+\alpha \frac{1}{n\left\|x_{k}\right\|_{2}} R_{r} x_{k}
$$

where $x_{k} \in R^{n}, \alpha$ is a positive number and becomes a rotation operator, $n$ denotes the number of variables, and $R_{r} \in R^{n \times n}$ is a uniformly distributed random matrix between $[-1,1]$. The operator is a search algorithm to search in a super surface with $x_{k}$ as the center and $\alpha$ as the radius, and the radius $\alpha$ satisfies $\left\|x_{k+1}-x_{k}\right\|_{2} \leq \alpha$.

Translation operator:

$$
x_{k+1}=x_{k}+\beta R_{t} \frac{x_{k}-x_{k-1}}{\left\|x_{k}-x_{k-1}\right\|_{2}}
$$

$\beta$ is a positive number and becomes a translation operator. $R_{t} \in R$ is a random number with a uniform distribution between $[0,1]$. The operator searches along the positive direction of the $x_{k-1}$ to $x_{k}$ gradient with a maximum search step of $\beta$.

Expansion operator:

$$
x_{k+1}=x_{k}+\gamma R_{e} x_{k}
$$


$\gamma$ is a positive number and becomes an expansion operator. $R_{e} \in R^{n \times n}$ is a diagonal matrix that follows a Gaussian distribution. The operator can optimize the whole search space.

Axesion operator:

$$
x_{k+1}=x_{k}+\delta R_{a} x_{k}
$$

$\delta$ is a positive number and becomes an axesion operator. $R_{a} \in R^{n \times n}$ is a diagonal matrix that follows a Gaussian distribution, and the matrix has only one random position with elements other than zero.

The basic flowchart of the STA is shown in Figure 1. In Figure 1, Best is the optimal individual in the species, $S E$ is the number of individuals in the search species, and $\alpha, \beta, \gamma, \delta$ are the rotation operator, translation operator, expansion operator, and axesion operator, respectively. In Figure 1, three steps of expansion, rotation, and axesion are performed, and the translation operation is embedded in these three steps.

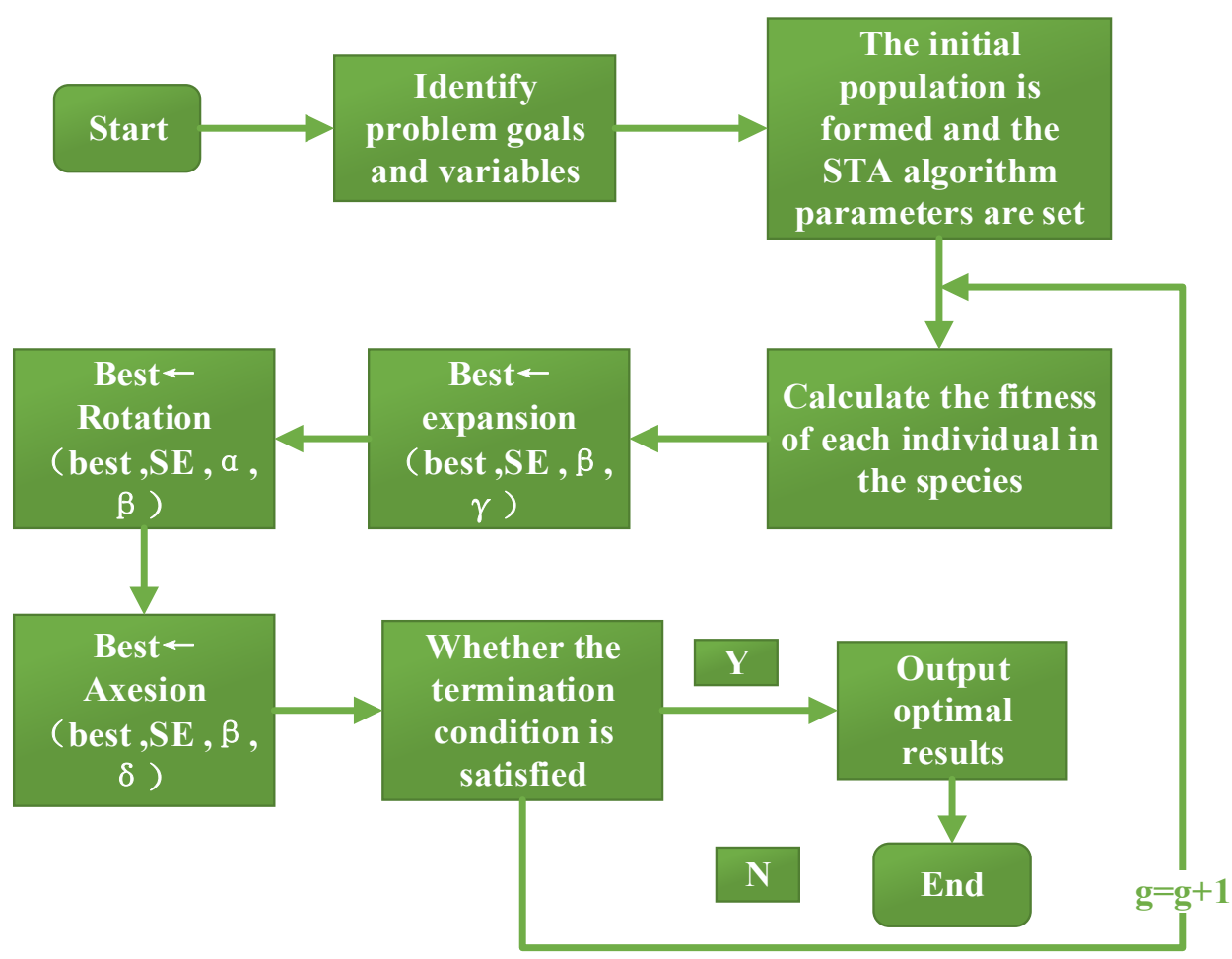

Figure 1. State transition algorithm (STA) flowchart.

Specific steps are as follows:

Step1: Determine the problem objectives and variables. The number of searches $S E$ for initializing the state transition algorithm and the value of each operation factor $\alpha, \beta, \gamma, \delta$.

Step2: Calculate the fitness value of each individual in the species.

Step3: $\quad$ Best $\leftarrow$ expansion $(B e s t, S E, \beta, \gamma)$, oldBest $\leftarrow$ Best, its fitness value is $f(B e s t)$.

Step4: Copy oldBest into a species with $S E$ individuals, and expand to obtain species state. Calculate the individual fitness value in state. At this time, the best individual in the group is newBest, and the fitness value is $g B e s t$.

Step5: If $g$ Best $<f($ Best) (for the minimization problem), then $f($ Best $) \leftarrow g B e s t$, Best $\leftarrow$ newBest, and execute Step6; otherwise, execute Step7.

Step6: $\quad x_{k-1} \leftarrow$ oldBest, $x_{k} \leftarrow$ Best, each copied SE times, paired in pairs. Species state is obtained after performing the transfer operation according to Equation (12). Calculate the individual fitness value in state. At this time, the optimal individual of the group is newBest, and the fitness value is $g$ Best. 
Step7: If $g$ Best $<f($ Best $)$, then $f($ Best $) \leftarrow g$ Best, Best $\leftarrow$ newBest .

Step8: Output Best as an expansion operation.

Step9: Best $\leftarrow$ rotation (Best, SE, $\alpha, \beta)$ and Best $\leftarrow$ axesion $(B e s t, S E, \beta, \delta)$ are similar to Step3-Step8.

Step10: Whether the final result satisfies the termination condition, if it is satisfied, the optimal result is output; otherwise, Step 2 to Step 9 are continued.

\subsection{Optimized ACMD Method}

Like other algorithms, the STA is based on the iterative search of individuals in the neighborhood. The difference is that each individual in the search species uses the group optimal value, that is, the elite individual. The search process is only related to the group optimal value of the previous generation, and the search is carried out within the group optimal neighborhood. For example, when performing the expansion operation, the optimal individual of the group is first taken as an input, the optimal individual is then copied to the number of individuals $S E$ of the search species, and the expansion operation is performed on these optimal individuals. The optimal individual in the group is calculated by performing the expansion operation, and the optimal individual is used as the input for the next operation.

The state transition algorithm has five important parameters, that is, the number of individuals in the search species $S E$, rotation operator $\alpha$, translation operator $\beta$, expansion operator $\gamma$, and axesion operator $\delta$. In the case where the number of individuals $S E$ is constant, the larger the value of $\alpha, \beta, \gamma, \delta$, the larger the search range of the corresponding operator, and the larger the search range, which is beneficial to the global search. When its value is small, the search accuracy is high, which is advantageous for the local search. In References [36,37], the species $S E$ is set to $S E=20$ and $S E=10$, respectively, because the number of artificial species will affect the accuracy of the search. This paper proposes the Rastrigin optimization test equation to optimize the number of different species, and detects the time of each optimization (the number of species is 1-100, in which the rotation operator, translation operator, expansion operator, and axesion operator are unchanged and are set to $\alpha=1, \beta=1, \gamma=1, \delta=1$, respectively). Through multiple inspections, the number of species of the state transition algorithm when the average optimization time is the shortest is finally determined.

Rastrigin function:

$$
f=\sum_{i=1}^{n}\left(x_{i}^{2}-10 \cos \left(2 \pi x_{i}\right)+10\right)[-5.12,5.12]
$$

where $x_{i}$ is the $i$-th element of the $n$-dimensional Euclid space element.

This paper uses the improved STA method to optimize in the ACMD method. The optimization process is shown in Figure 2.

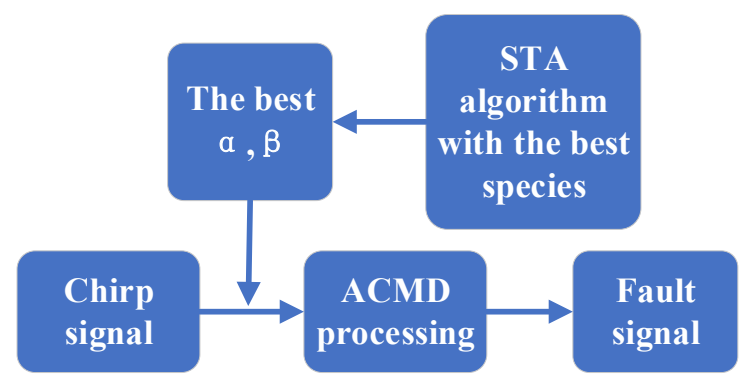

Figure 2. Optimized adaptive chirp mode decomposition (ACMD) method.

Step1: Determine the species of the STA when the average optimization time is the shortest through multiple detections.

Step2: Bring the number of optimal species groups into the STA algorithm.

Step3: Employ the optimized STA algorithm to find the optimal parameter. 
Step4: The optimal parameter is input into ACMD, and the optimized ACMD is used to process the chirp signal.

\section{Simulation}

\subsection{STA}

This article applies the intelligent algorithms of Ant Lion Optimizer (ALO), Grey Wolf Optimizer, Lightning Search Algorithm (LSA), Particle Swarm Optimization (PSO), STA, and Whale Optimization Algorithm (WOA) to the optimization process of Rastrigin optimization test equations. The number of functions is set to 100 , the number of iterations is set to 500 , and the operation is repeated 30 times. The total running time is shown in Table 1. The fastest speed of the STA algorithm is $13.196 \mathrm{~s}$.

Table 1. The intelligent algorithm optimization time.

\begin{tabular}{cr}
\hline Intelligent Algorithm & Times \\
\hline Ant Lion Optimizer (ALO) & $17.741 \mathrm{~s}$ \\
Grey Wolf Optimizer & $19.915 \mathrm{~s}$ \\
Lightning Search Algorithm (LSA) & $13.715 \mathrm{~s}$ \\
Particle Swarm Optimization (PSO) & $19.781 \mathrm{~s}$ \\
STA & $13.196 \mathrm{~s}$ \\
Whale Optimization Algorithm (WOA) & $18.547 \mathrm{~s}$ \\
\hline
\end{tabular}

Because the number of species of STA affects the speed and accuracy of the algorithm, before the study in this paper, the species number was set artificially and there were human errors. This paper proposes the Rastrigin optimization test equation to optimize the number of different species, and detects the time of each optimization (the number of species is $1-100$, in which the rotation operator, translation operator, expansion operator, and axesion operator are unchanged and are set to $\alpha=1, \beta=1, \gamma=1, \delta=1$, respectively) and obtains the result shown in Figure 3. In Figure 3, the $\mathrm{X}$-coordinate is the number of different species, and the $\mathrm{Y}$-coordinate is the optimization time. After three runs, it is clearly observed in Figure 3 that the number of optimal species for the first run was 32, and the optimization time was $0.05793 \mathrm{~s}$. The number of optimal species in the second run was 9 , and the optimization time was $0.06298 \mathrm{~s}$. The number of optimal species in the third run was 36 , and the optimization time was $0.06833 \mathrm{~s}$.

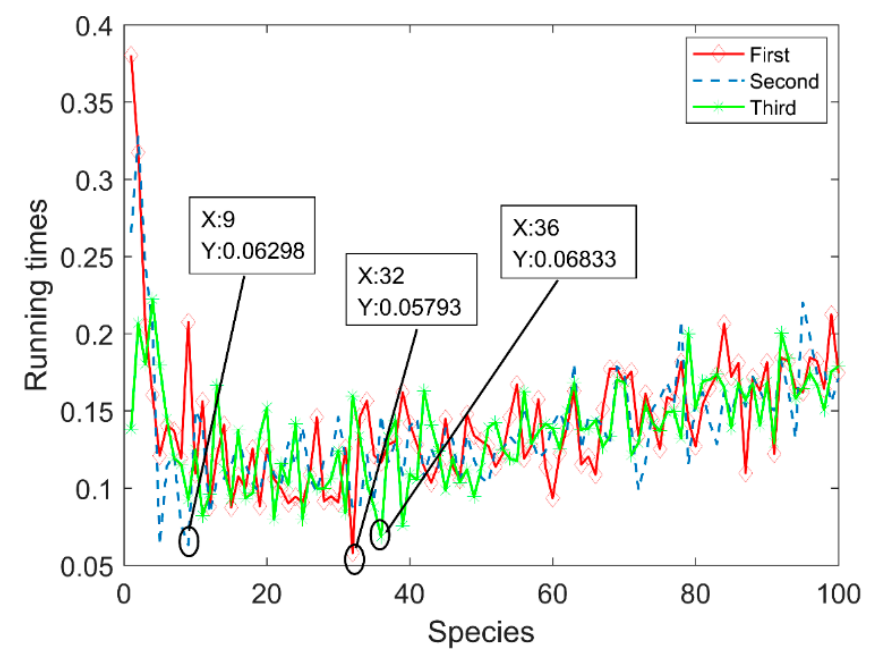

Figure 3. Different species running times.

It can be predicted that the optimal number of species is different in each run, so in order to determine the optimal number of species more accurately, this paper will optimize each species 
10,000 times and record the time of each run. As depicted in Figure 3, the number of optimal species groups is uncertain for each run. In order to determine the species number accurately, this paper takes the average value of 10,000 times the running time of the same species optimization. Then, 1-100 species are taken and run 10,000 times to give Figure 4. The X-coordinate is the number of species and the Y-coordinate is the average running time of 10,000 times for the same species optimization. Figure 4 clearly shows that when the species is $S E=9$, the shortest average optimization time is $0.09718 \mathrm{~s}$. Compared to the artificially set species, the method proposed in this paper can improve the efficiency of optimization and save time. This article takes $S E=9, S E=10, S E=20$, and $S E=30$ as examples to run each species 10,000 times, and records the time of each run to give Figure 5, where the $\mathrm{X}$-coordinate is the number of runs and the Y-coordinate is the running time. In Figure $5 \mathrm{a}$, when the species is $S E=9$, the change in the running time is relatively uniform, and there is no sudden change. Figure $5 b-d$ show that the time amplitude of each optimization of the same species greatly changes. Therefore, taking $S E=9$ can improve the efficiency of optimization and save time for optimization.

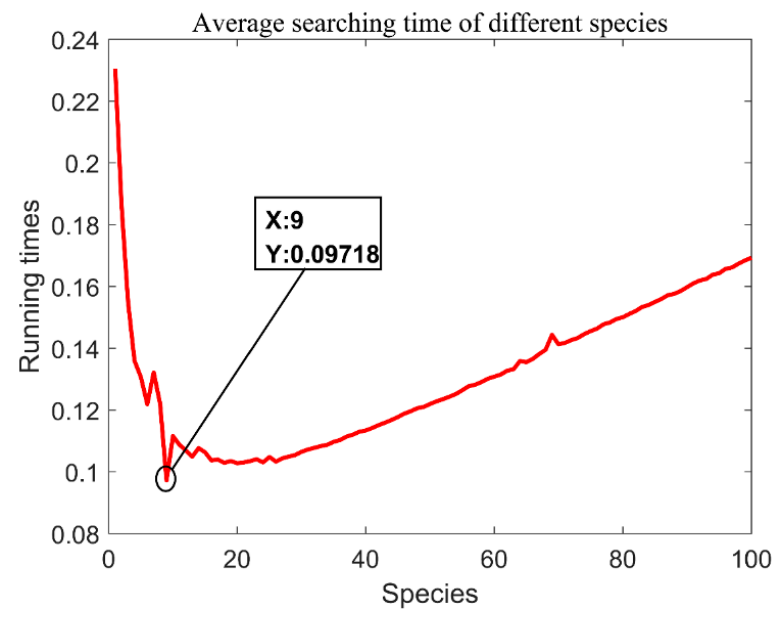

Figure 4. The average running time of different species.

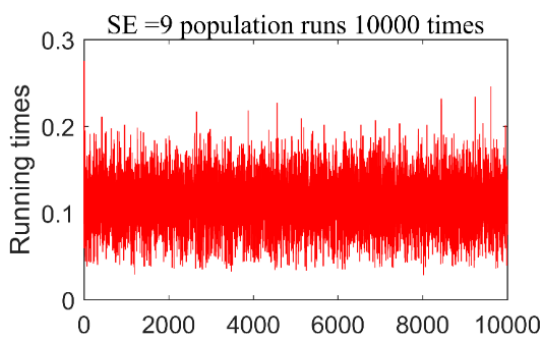

(a)

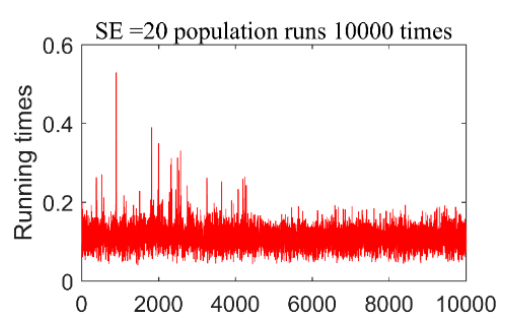

(c)

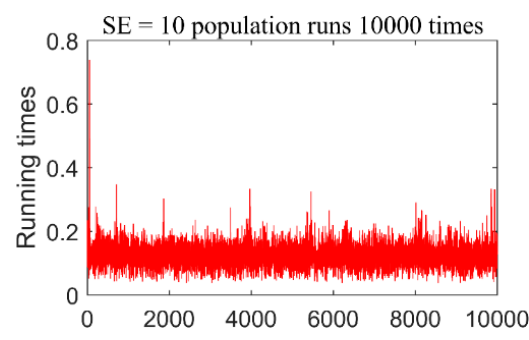

(b)

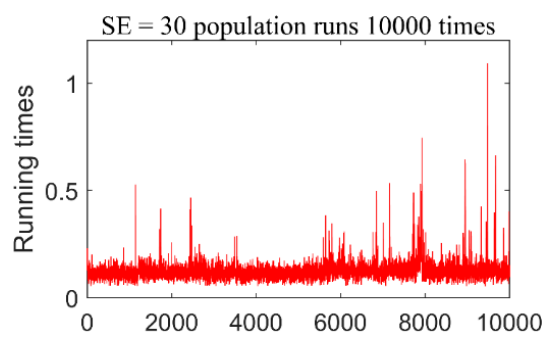

(d)

Figure 5. Different species run 10,000 times ((a). SE $=9$ population runs 10,000 times. (b). $\mathrm{SE}=10$ population runs 10,000 times. (c). $\mathrm{SE}=20$ population runs 10,000 times. (d). SE $=30$ population runs 10,000 times). 


\subsection{Parameter Optimization}

In order to accurately compare with ACMD, it is proved that the method proposed in this paper optimizes the $\alpha, \beta$ value in the ACMD. In this paper, the same simulation signal is constructed as ACMD (two signals with large IFs fluctuation):

$$
\left\{\begin{array}{l}
S_{1}(t)=\exp (-0.3 t) \times \cos (700 \pi t+\cos (50 \pi t)) \\
S_{2}(t)=\exp (-0.6 t) \times \cos (500 \pi t+\cos (40 \pi t)) \\
S(t)=S_{1}(t)+S_{2}(t)
\end{array}\right.
$$

The two signals IFs are $f_{1}(t)=350-25 \sin (50 \pi t)$ and $f_{2}(t)=250-20 \sin (40 \pi t)$, respectively. The time-domain and frequency-domain diagrams of the signal are shown in Figure 6. The two main frequencies in the spectrum $(250$ and $350 \mathrm{~Hz})$ correspond to the center frequencies of the IFs of the two signal components.

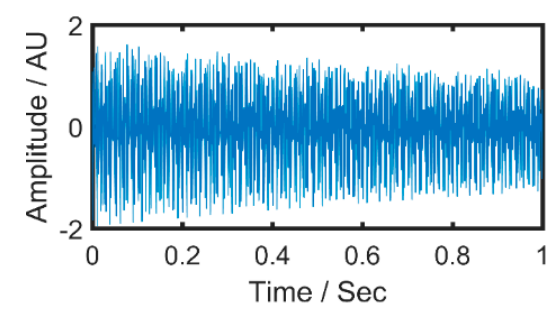

(a)

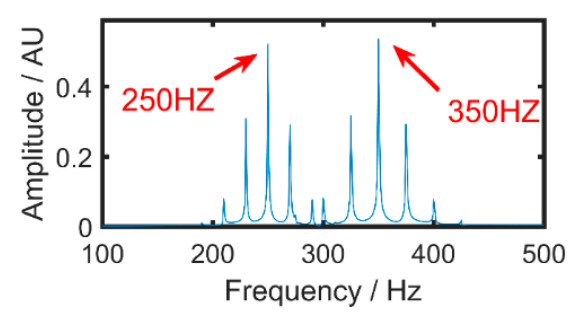

(b)

Figure 6. Simulation signal ((a) time-domain diagram, (b) frequency domain diagram).

$\alpha$ and $\beta$ of the ACMD processing simulation signal are set to $1 \times 10^{-3}$ and $1 \times 10^{-4}$, respectively. The simulated signals were processed by ACMD to obtain the final estimated signal IFs. The SNRs of IFs1 and $\mathrm{S} 1(\mathrm{t})$, and IFs2 and S2 (t) are calculated, respectively, and SNR1 = 23.970, SNR2 = 29.155.

An improved STA was used to optimize $\alpha$ and $\beta$ in ACMD, with the SNR as the Z-axis, and C and $\mathrm{D}$ as the $\mathrm{X}$ - and Y-axes, respectively. The results are shown in Figure 7. The range of the $\alpha$ value optimization in this paper is $1 \times 10^{-5}-1 \times 10^{-2}$, and the range of $\beta$ value optimization in this paper is $1 \times 10^{-6}-1 \times 10^{-3}$. In Figure $7 \mathrm{a}, S N R 1=26.16, \alpha=3 \times 10^{-5}, \beta=8.61 \times 10^{-4}$. In Figure $7 \mathrm{~b}$, SNR2 $=32.63, \alpha=6 \times 10^{-5}$, and $\beta=2.95 \times 10^{-4}$. As there are $10^{6}$ data in the optimization result, the color display of the graph is not obvious, so this paper extracts 400 data points around the largest data point to enlarge the graph. As shown in Figure $7 \mathrm{a}, \mathrm{b}$, the maximum point can be clearly observed in the figure.

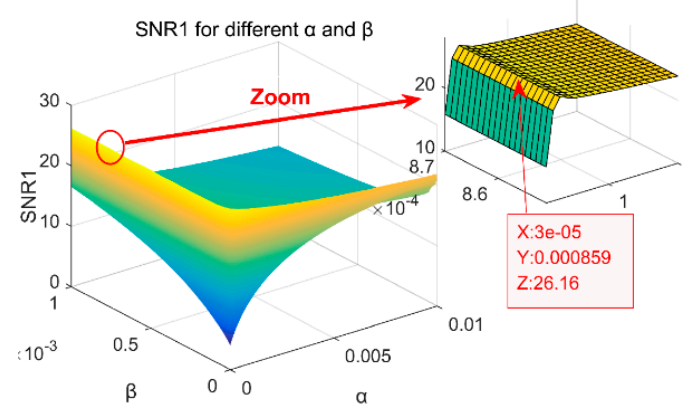

(a)

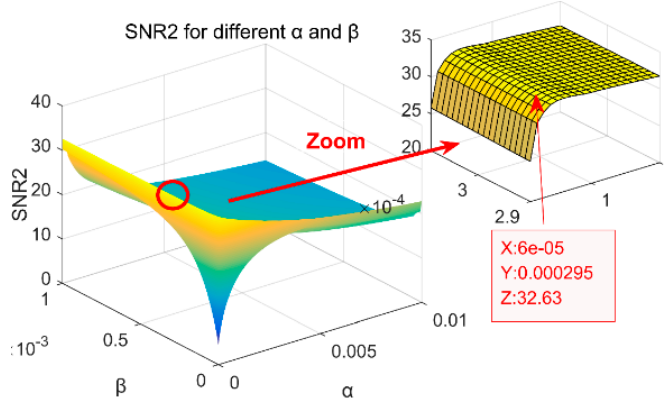

(b)

Figure 7. Instantaneous frequency(IF)s1 and IFs2 optimization process((a). SNR1 for different $\alpha$ and $\beta$; (b). SNR2 for different $\alpha$ and $\beta$ ). 
The figure in this paper is the simulation display of Matlab2016a version, and the data in Figure 7a,b are the approximations obtained by rounding. This article exports 100 detailed data, as shown in Tables 2 and 3 , where Table 2 is the IFs 1 with different $\alpha, \beta$ corresponding to SNR data. Table 3 is the IFs 2 with different $\alpha, \beta$ corresponding to SNR data. It can be observed from Table 2 that when $S N R 1=26.159$, the SNR of IFs1 is the largest. When SNR2 $=32.633$, the SNR of IFs2 is the largest in Table 3.

Table 2. IFs1 with different $\alpha, \beta$ corresponding to signal-to-noise ratio (SNR) data.

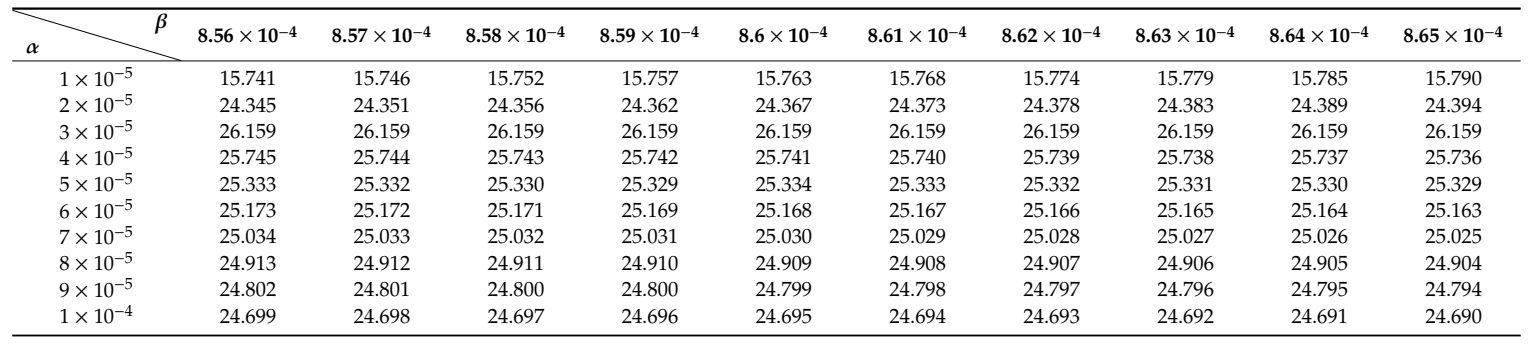

Table 3. IFs2 with different $\alpha, \beta$ corresponding to SNR data.

\begin{tabular}{|c|c|c|c|c|c|c|c|c|c|c|}
\hline$\alpha$ & $2.9 \times 10^{-4}$ & $2.91 \times 10^{-4}$ & $2.92 \times 10^{-4}$ & $2.93 \times 10^{-4}$ & $2.94 \times 10^{-4}$ & $2.95 \times 10^{-4}$ & $2.96 \times 10^{-4}$ & $2.97 \times 10^{-4}$ & $2.98 \times 10^{-4}$ & $2.99 \times 10^{-4}$ \\
\hline $1 \times 10^{-5}$ & 25.424 & 25.445 & 25.465 & 25.486 & 25.506 & 25.526 & 25.546 & 25.566 & 25.586 & 25.606 \\
\hline $2 \times 10^{-5}$ & 30.307 & 30.319 & 30.332 & 30.332 & 30.344 & 30.357 & 30.356 & 30.369 & 30.381 & 30.381 \\
\hline $3 \times 10^{-5}$ & 31.565 & 31.560 & 31.572 & 31.584 & 31.595 & 31.590 & 31.602 & 31.613 & 31.625 & 31.619 \\
\hline $4 \times 10^{-5}$ & 32.270 & 32.279 & 32.268 & 32.277 & 32.285 & 32.294 & 32.303 & 32.291 & 32.299 & 32.308 \\
\hline $5 \times 10^{-5}$ & 32.563 & 32.569 & 32.574 & 32.580 & 32.564 & 32.570 & 32.575 & 32.580 & 32.586 & 32.591 \\
\hline $6 \times 10^{-5}$ & 32.619 & 32.622 & 32.625 & 32.628 & 32.630 & 32.633 & 32.616 & 32.619 & 32.621 & 32.624 \\
\hline $7 \times 10^{-5}$ & 32.569 & 32.570 & 32.571 & 32.573 & 32.555 & 32.556 & 32.558 & 32.559 & 32.560 & 32.561 \\
\hline $8 \times 10^{-5}$ & 32.465 & 32.465 & 32.465 & 32.448 & 32.448 & 32.448 & 32.448 & 32.448 & 32.448 & 32.447 \\
\hline $9 \times 10^{-5}$ & 32.333 & 32.332 & 32.331 & 32.330 & 32.330 & 32.314 & 32.313 & 32.312 & 32.311 & 32.310 \\
\hline $1 \times 10^{-4}$ & 32.276 & 32.281 & 32.266 & 32.271 & 32.270 & 32.269 & 32.274 & 32.273 & 32.278 & 32.277 \\
\hline
\end{tabular}

Based on the above chart, we can obtain such a conclusion: When the SNR of different signals is the largest, they correspond to different $\alpha$ and $\beta$ values. Therefore, ACMD uses artificial settings of $\alpha$ and $\beta$, and using the same $\alpha$ and $\beta$ for different signals has drawbacks. Employing the method proposed in this paper can improve the SNR. The SNR improvement is as follows:

$$
R=\frac{S N R_{i}-S N R_{o}}{S N R_{o}} \times 100 \%
$$

where $R$ is the SNR improvement; $S N R_{i}$ denotes the SNR of the method proposed in this article; $S N R_{o}$ denotes the SNR obtained using the ACMD method.

From Equation (17), we can see that the IFs1 and IFs2 obtained by the method proposed in this paper are $9.134 \%$ and $11.931 \%$ higher than those obtained by the ACMD method, respectively.

\section{Experimental Verification}

In the experimental part, the proposed method was applied to the signals from the marine animals False Killer Whale, Baird's Beaked Whale, Weddell Seal, and Bottlenose Dolphin [38]. As marine animals use sound for echolocation and communication, it is very important to analyze these sound signals in studying the behavior of marine animals. As shown in Figure 8, the time-domain diagram of the animal sound signal and the time-frequency diagram processed by the ACMD method are shown, where $\alpha=1 \times 10^{-3}, \beta=1 \times 10^{-4}$. It can be observed from the time-frequency diagram that there is noise interference in the signal. 

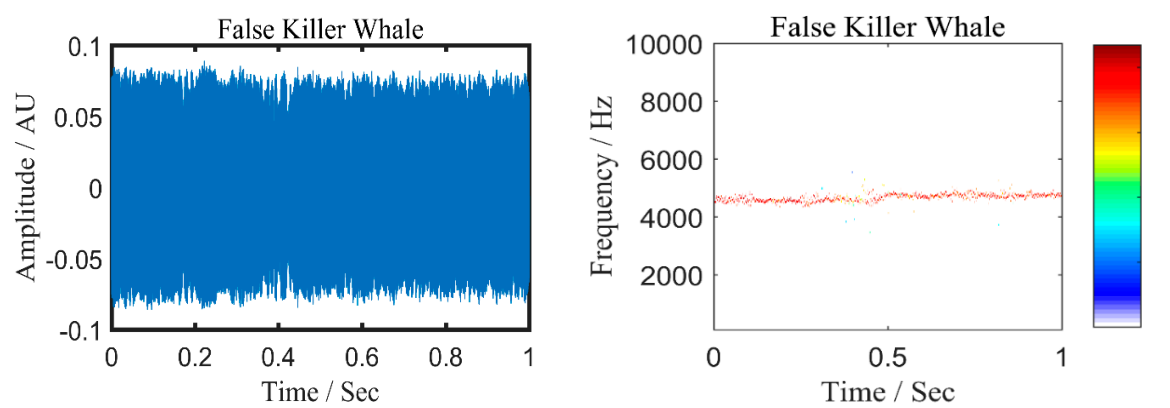

(a)
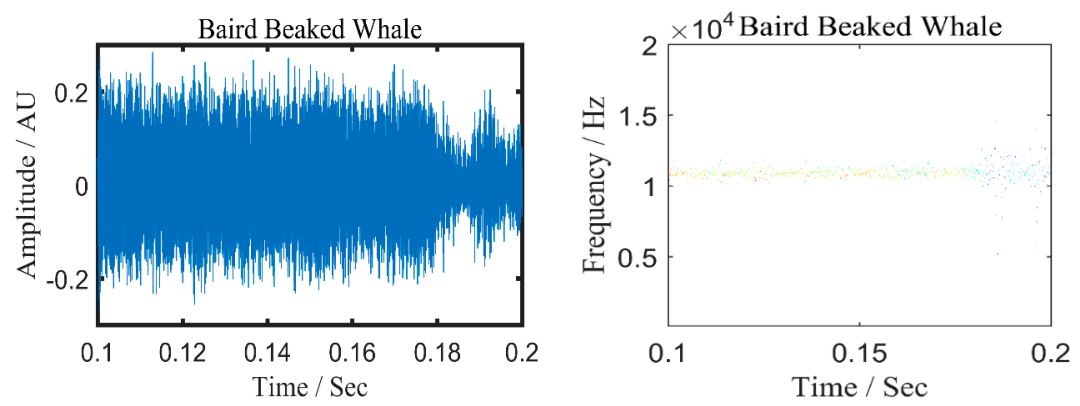

(b)
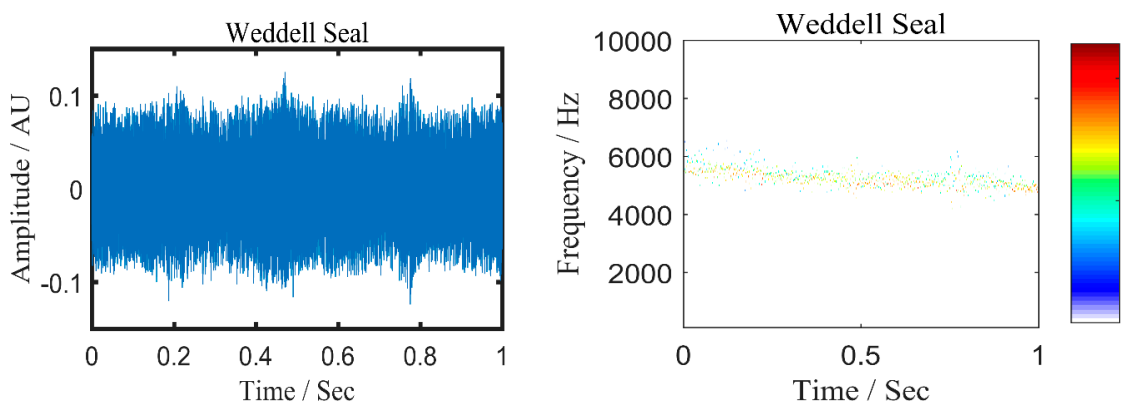

(c)
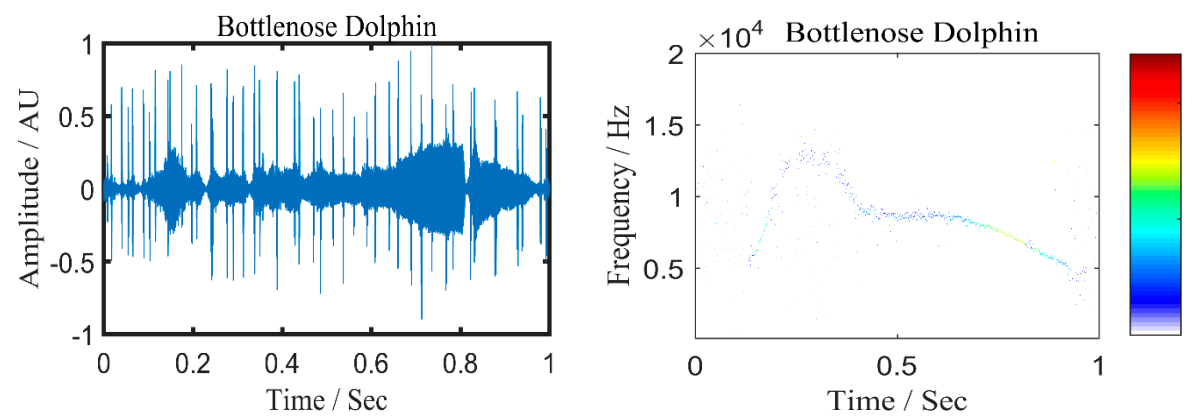

(d)

Figure 8. Four kinds of animal sound signals and the time-domain diagram processed by ACMD method ((a). The time-domain diagram of the False Killer Whale sound signal and the time-frequency diagram processed by the ACMD method a, where $\alpha=1 \times 10^{-3}, \beta=1 \times 10^{-4}$; (b). The time-domain diagram of the Baird's Beaked Whale sound signal and the time-frequency diagram processed by the ACMD method a, where $\alpha=1 \times 10^{-3}, \beta=1 \times 10^{-4}$; (c). The time-domain diagram of the Weddell Seal sound signal and the time-frequency diagram processed by the ACMD method a, where $\alpha=1 \times 10^{-3}, \beta=1 \times 10^{-4} ;(\mathbf{d})$. The time-domain diagram of the Bottlenose Dolphin sound signal and the time-frequency diagram processed by the ACMD method a, where $\alpha=1 \times 10^{-3}, \beta=1 \times 10^{-4}$ ). 
The method proposed in this article is employed to find the optimal $\alpha, \beta$ value, and its optimization result is shown in Figure 9. Due to a large amount of data, this article will locally enlarge around its maximum point of SNR. The $\alpha, \beta$ corresponding to the maximum point of the four types of biological SNR in the Figure 10 is: False Killer Whale, $\alpha=3.26 \times 10^{-3}, \beta=1 \times 10^{-6}$; Baird's Beaked Whale, $\alpha=9.96 \times 10^{-3}, \beta=9.96 \times 10^{-4}$; Weddell Seal, $\alpha=9.96 \times 10^{-3}, \beta=9.96 \times 10^{-4}$; Bottlenose Dolphin, $\alpha=9.86 \times 10^{-3}, \beta=9.76 \times 10^{-4}$. The most suitable $\alpha, \beta$ is brought into ACMD and the marine animals' signal is processed to obtain the results shown in Figure 10. Compared to the time-frequency diagram in Figures 8 and 10, it can be observed that the signal noise in Figure 10 is less, which proves the effectiveness of the method proposed in this paper.

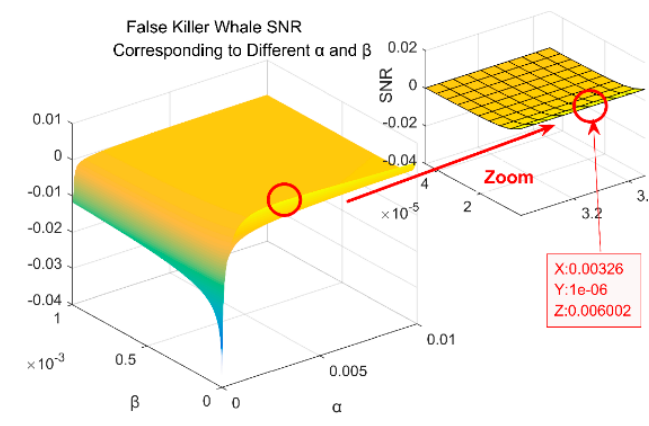

(a)

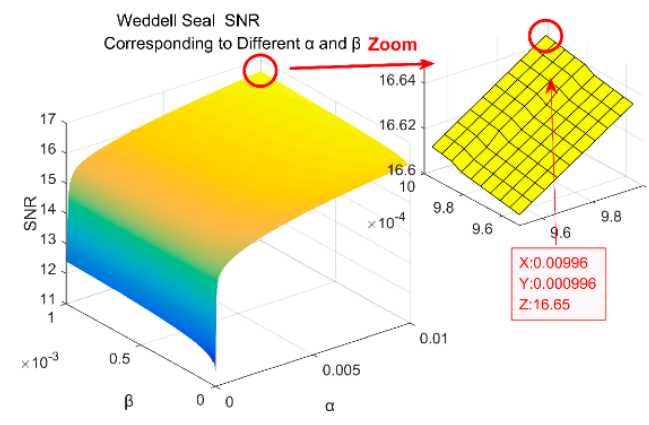

(c)

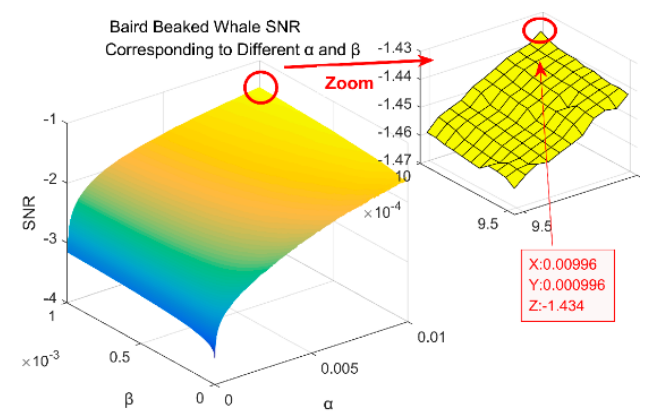

(b)

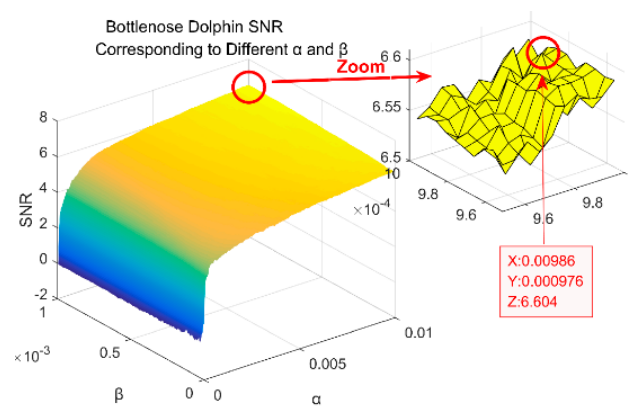

(d)

Figure 9. The most suitable $\alpha$ and $\beta$ for four kinds of animals ((a). False Killer Whale's locally enlarge around maximum point of SNR; (b). Baird's Beaked Whale's locally enlarge around maximum point of SNR; (c) Weddell Seal's locally enlarge around maximum point of SNR; (d) Bottlenose Dolphin's locally enlarge around maximum point of SNR).

In order to describe the change in the SNR before and after improvement, this paper uses Equation (17) to show the change in the SNR before and after. According to Equation (17), the SNRs of the False Killer Whale, Baird's Beaked Whale, Weddell Seal, and Bottlenose Dolphin that increased are $672.018 \%,-185.595 \%, 7.777 \%$, and $32.759 \%$, respectively. Table 4 lists the SNR changes before and after ACMD optimization. Although the improvement in Baird's beaked whale is $-185.595 \%$; in fact, the SNR is increased from -2.443 to -1.434 . In Tables 5-8, 100 SNR parameters around the maximum SNR of four marine animals are listed. 


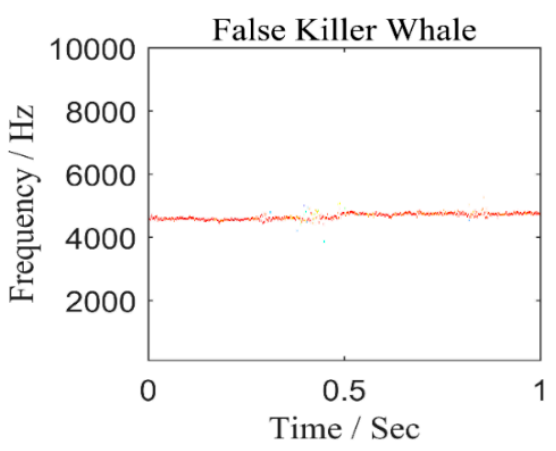

(a)

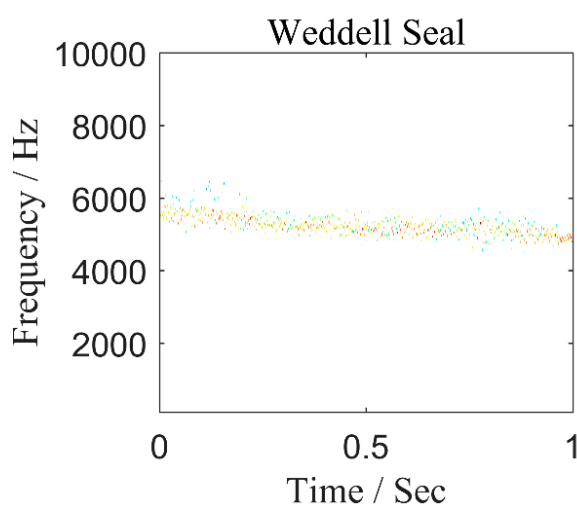

(c)

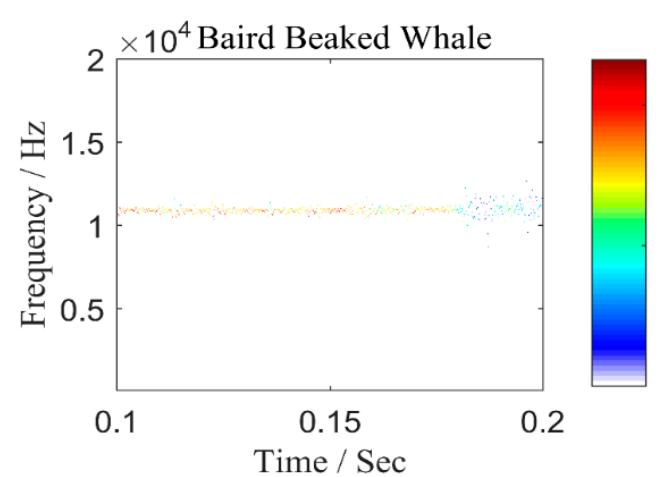

(b)

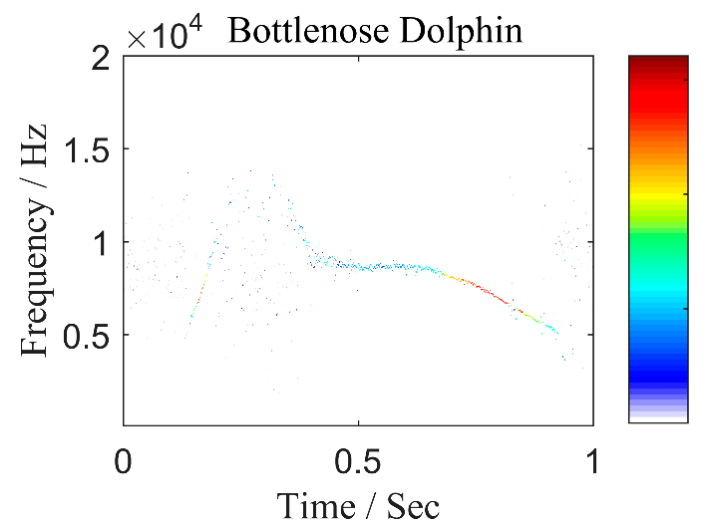

(d)

Figure 10. Optimized ACMD processing animal sound signals. (a) $\alpha=3.26 \times 10^{-3}, \beta=1 \times 10^{-6}$, (b) $\alpha=9.96 \times 10^{-3}, \beta=9.96 \times 10^{-4}$, (c) $\alpha=9.96 \times 10^{-3}, \beta=9.96 \times 10^{-4}$, (d) $\alpha=9.86 \times 10^{-3}, \beta=9.76 \times 10^{-4}$.

Table 4. SNR changes before and after ACMD optimization.

\begin{tabular}{cccc}
\hline Marine Animal & ACMD & Optimize ACMD & Increase \\
\hline False Killer Whale & $\alpha=1 \times 10^{-3}, \beta=1 \times 10^{-4}$ & $\alpha=3.26 \times 10^{-3}, \beta=1 \times 10^{-6}$ & $672.018 \%$ \\
Baird's Beaked Whale & $\alpha=1 \times 10^{-3}, \beta=1 \times 10^{-4}$ & $\alpha=9.96 \times 10^{-3}, \beta=9.96 \times 10^{-4}$ & $-185.595 \%$ \\
Weddell Seal & $\alpha=1 \times 10^{-3}, \beta=1 \times 10^{-4}$ & $\alpha=9.96 \times 10^{-3}, \beta=9.96 \times 10^{-4}$ & $7.777 \%$ \\
Bottlenose Dolphin & $\alpha=1 \times 10^{-3}, \beta=1 \times 10^{-4}$ & $\alpha=9.86 \times 10^{-3}, \beta=9.76 \times 10^{-4}$ & $32.759 \%$ \\
\hline
\end{tabular}

Table 5. False Killer Whale with different $\alpha, \beta$ corresponding to SNR data.

\begin{tabular}{|c|c|c|c|c|c|c|c|c|c|c|}
\hline$\beta$ & $1 \times 10^{-6}$ & $6 \times 10^{-6}$ & $1.1 \times 10^{-5}$ & $1.6 \times 10^{-5}$ & $2.1 \times 10^{-5}$ & $2.6 \times 10^{-5}$ & $3.1 \times 10^{-5}$ & $3.6 \times 10^{-5}$ & $4.1 \times 10^{-5}$ & $4.6 \times 10^{-5}$ \\
\hline $3.01 \times 10^{-3}$ & 0.0059 & 0.0028 & 0.0014 & 0.0009 & 0.0006 & 0.0004 & 0.0003 & 0.0003 & 0.0002 & 0.0002 \\
\hline $3.06 \times 10^{-3}$ & 0.0059 & 0.0029 & 0.0015 & 0.0009 & 0.0006 & 0.0005 & 0.0004 & 0.0003 & 0.0003 & 0.0002 \\
\hline $3.11 \times 10^{-3}$ & 0.0059 & 0.0029 & 0.0015 & 0.0010 & 0.0007 & 0.0005 & 0.0004 & 0.0003 & 0.0003 & 0.0002 \\
\hline $3.16 \times 10^{-3}$ & 0.0059 & 0.0029 & 0.0015 & 0.0010 & 0.0007 & 0.0005 & 0.0004 & 0.0003 & 0.0003 & 0.0003 \\
\hline $3.21 \times 10^{-3}$ & 0.0059 & 0.0030 & 0.0016 & 0.0010 & 0.0007 & 0.0005 & 0.0004 & 0.0004 & 0.0003 & 0.0003 \\
\hline $3.26 \times 10^{-3}$ & 0.0060 & 0.0030 & 0.0016 & 0.0010 & 0.0007 & 0.0006 & 0.0005 & 0.0004 & 0.0003 & 0.0003 \\
\hline $3.31 \times 10^{-3}$ & 0.0059 & 0.0030 & 0.0017 & 0.0011 & 0.0008 & 0.0006 & 0.0005 & 0.0004 & 0.0004 & 0.0003 \\
\hline $3.36 \times 10^{-3}$ & 0.0059 & 0.0031 & 0.0017 & 0.0011 & 0.0008 & 0.0006 & 0.0005 & 0.0004 & 0.0004 & 0.0003 \\
\hline $3.41 \times 10^{-3}$ & 0.0059 & 0.0031 & 0.0017 & 0.0011 & 0.0008 & 0.0006 & 0.0005 & 0.0004 & 0.0004 & 0.0003 \\
\hline $3.46 \times 10^{-3}$ & 0.0059 & 0.0031 & 0.0017 & 0.0011 & 0.0008 & 0.0006 & 0.0005 & 0.0004 & 0.0004 & 0.0003 \\
\hline
\end{tabular}


Table 6. Beaked Whale with different $\alpha, \beta$ corresponding to SNR data.

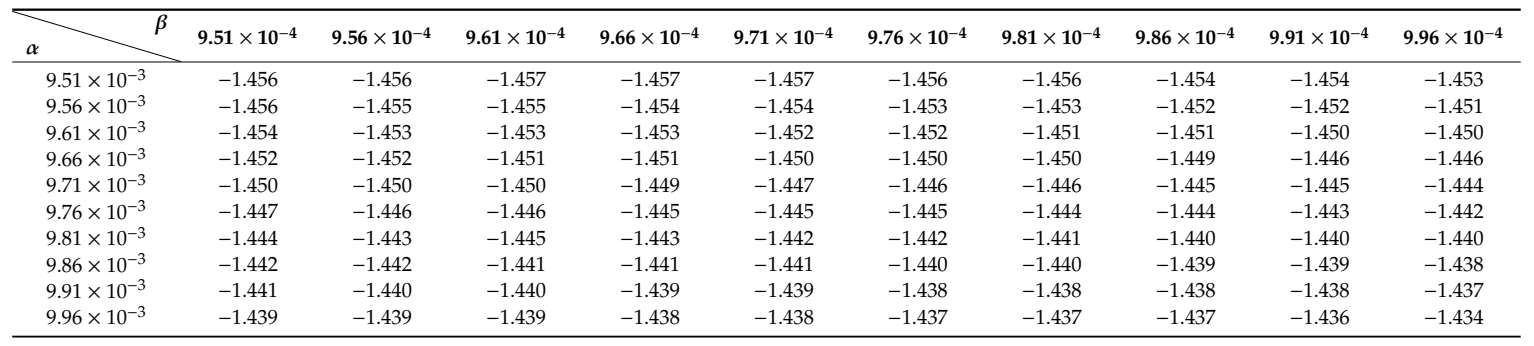

Table 7. Weddell Seal with different $\alpha, \beta$ corresponding to SNR data.

\begin{tabular}{|c|c|c|c|c|c|c|c|c|c|c|}
\hline${ }_{\alpha} \quad \beta$ & $9.51 \times 10^{-4}$ & $9.56 \times 10^{-4}$ & $9.61 \times 10^{-4}$ & $9.66 \times 10^{-4}$ & $9.71 \times 10^{-4}$ & $9.76 \times 10^{-4}$ & $9.81 \times 10^{-4}$ & $9.86 \times 10^{-4}$ & $9.91 \times 10^{-4}$ & $9.96 \times 10^{-4}$ \\
\hline $9.51 \times 10^{-3}$ & 16.604 & 16.605 & 16.606 & 16.607 & 16.607 & 16.608 & 16.609 & 16.612 & 16.612 & 16.613 \\
\hline $9.56 \times 10^{-3}$ & 16.608 & 16.609 & 16.609 & 16.610 & 16.611 & 16.612 & 16.613 & 16.615 & 16.616 & 16.617 \\
\hline $9.61 \times 10^{-3}$ & 16.612 & 16.612 & 16.613 & 16.614 & 16.615 & 16.616 & 16.616 & 16.619 & 16.620 & 16.621 \\
\hline $9.66 \times 10^{-3}$ & 16.615 & 16.616 & 16.617 & 16.618 & 16.619 & 16.619 & 16.622 & 16.623 & 16.624 & 16.624 \\
\hline $9.71 \times 10^{-3}$ & 16.619 & 16.620 & 16.621 & 16.621 & 16.622 & 16.623 & 16.626 & 16.626 & 16.627 & 16.628 \\
\hline $9.76 \times 10^{-3}$ & 16.623 & 16.623 & 16.624 & 16.625 & 16.626 & 16.627 & 16.629 & 16.630 & 16.631 & 16.632 \\
\hline $9.81 \times 10^{-3}$ & 16.626 & 16.627 & 16.628 & 16.629 & 16.630 & 16.630 & 16.633 & 16.634 & 16.635 & 16.636 \\
\hline $9.86 \times 10^{-3}$ & 16.630 & 16.631 & 16.632 & 16.632 & 16.633 & 16.636 & 16.637 & 16.638 & 16.638 & 16.639 \\
\hline $9.91 \times 10^{-3}$ & 16.634 & 16.634 & 16.635 & 16.636 & 16.637 & 16.640 & 16.640 & 16.641 & 16.642 & 16.643 \\
\hline $9.96 \times 10^{-3}$ & 16.637 & 16.638 & 16.639 & 16.640 & 16.641 & 16.643 & 16.644 & 16.645 & 16.646 & 16.647 \\
\hline
\end{tabular}

Table 8. Bottlenose Dolphin with different $\alpha, \beta$ corresponding to SNR data.

\begin{tabular}{|c|c|c|c|c|c|c|c|c|c|c|}
\hline$\beta$ & $9.51 \times 10^{-4}$ & $9.56 \times 10^{-4}$ & $9.61 \times 10^{-4}$ & $9.66 \times 10^{-4}$ & $9.71 \times 10^{-4}$ & $9.76 \times 10^{-4}$ & $9.81 \times 10^{-4}$ & $9.86 \times 10^{-4}$ & $9.91 \times 10^{-4}$ & $9.96 \times 10^{-4}$ \\
\hline $9.51 \times 10^{-3}$ & 6.546 & 6.555 & 6.551 & 6.539 & 6.542 & 6.545 & 6.548 & 6.553 & 6.553 & 6.545 \\
\hline $9.56 \times 10^{-3}$ & 6.547 & 6.527 & 6.538 & 6.543 & 6.545 & 6.548 & 6.538 & 6.551 & 6.550 & 6.553 \\
\hline $9.61 \times 10^{-3}$ & 6.561 & 6.544 & 6.549 & 6.562 & 6.551 & 6.558 & 6.566 & 6.556 & 6.566 & 6.569 \\
\hline $9.66 \times 10^{-3}$ & 6.547 & 6.529 & 6.554 & 6.528 & 6.550 & 6.559 & 6.545 & 6.550 & 6.560 & 6.567 \\
\hline $9.71 \times 10^{-3}$ & 6.558 & 6.542 & 6.546 & 6.551 & 6.552 & 6.555 & 6.549 & 6.559 & 6.548 & 6.548 \\
\hline $9.76 \times 10^{-3}$ & 6.584 & 6.579 & 6.582 & 6.585 & 6.579 & 6.581 & 6.580 & 6.554 & 6.557 & 6.564 \\
\hline $9.81 \times 10^{-3}$ & 6.591 & 6.566 & 6.590 & 6.588 & 6.592 & 6.587 & 6.565 & 6.581 & 6.579 & 6.558 \\
\hline $9.86 \times 10^{-3}$ & 6.584 & 6.579 & 6.589 & 6.590 & 6.580 & 6.604 & 6.591 & 6.567 & 6.594 & 6.578 \\
\hline $9.91 \times 10^{-3}$ & 6.582 & 6.565 & 6.591 & 6.588 & 6.589 & 6.590 & 6.592 & 6.573 & 6.550 & 6.576 \\
\hline $9.96 \times 10^{-3}$ & 6.594 & 6.593 & 6.595 & 6.596 & 6.585 & 6.594 & 6.595 & 6.591 & 6.576 & 6.589 \\
\hline
\end{tabular}

\section{Conclusions}

The ACMD method has a good effect on processing chirp signals. The parameter $\alpha$ controls the smoothness of the output signal. Too small an $\alpha$ will cause a smooth output signal. The parameter $\beta$ controls the IFs. If too small a $\beta$ is used, the output IF will be very smooth. However, rapidly changing IFs require relatively large $\beta$. The choice of $\alpha, \beta$ is artificially set, and there are errors in practical applications. Therefore, it employs STA to adaptively optimize $\alpha, \beta$. As the number of species in the STA is an artificial error, this paper proposes the Rastrigin optimization test equation to optimize the number of different species. Finally, the ACMD method optimized in this paper is applied to the chirp signal from marine animals, which verifies the feasibility of the proposed method. The following conclusions can be drawn in this paper:

1. This article applies the intelligent algorithms of Ant Lion Optimizer (ALO), Grey Wolf Optimizer, Lightning Search Algorithm (LSA), Particle Swarm Optimization (PSO), STA, and Whale Optimization Algorithm (WOA) to the optimization process of Rastrigin optimization test equations. The comparison shows that the fastest STA is $13.196 \mathrm{~s}$. Through a large number of simulation experiments, it is learned that when the population is 9, the STA algorithm runs fastest.

2. The artificial $\alpha, \beta$ in ACMD has an error when processing the signal, and different signals require different $\alpha, \beta$ values. 
Author Contributions: W.D. and N.D. performed the simulation experiment; J.W., Z.W. and Z.Z. analyzed the data and contributed reagents/materials/analysis tools; H.H. wrote the paper. All authors have read and agreed to the published version of the manuscript.

Funding: This work was supported by the National Natural Science Foundation of China (51905496), Shanxi Applied Basic Research Project (Grant No. 201801D221237), the project supported by Science Foundation of North University of China (NO. XJJ201802), the Open Fund of Key Laboratory of Advanced Manufacturing Technology of Shanxi Province (XJZZ201802).

Conflicts of Interest: The authors declare no conflict of interest.

\section{References}

1. Baroni, M. Linguistic generalization and compositionality in modern artificial neural networks. Philos. Trans. R. Soc. B-Biol. Sci. 2020, 375, 20190307. [CrossRef] [PubMed]

2. Lee, H.; Roan, M.J.; Ming, C.; Simmons, J.A.; Wang, R.; Muller, R. High-frequency soundfield microphone for the analysis of bat biosonar. J. Acoust. Soc. Am. 2019, 146, 4525. [CrossRef] [PubMed]

3. Pedersen, M.B.; Fahlman, A.; Borque-Espinosa, A.; Madsen, P.T.; Jensen, F.H. Whistling is metabolically cheap for communicating bottlenose dolphins (Tursiops truncatus). J. Exp. Biol. 2020, 223, jeb212498. [CrossRef] [PubMed]

4. Allen, J.A.; Garland, E.C.; Dunlop, R.A.; Noad, M.J. Network analysis reveals underlying syntactic features in a vocally learnt mammalian display, humpback whale song. Proc. R. Soc. B-Biol. Sci. 2019, 286, 20192014. [CrossRef]

5. Coluccia, A.; Fascista, A.; Ricci, G. A novel approach to robust radar detection of range-spread targets. Signal Process. 2020, 166, 107223. [CrossRef]

6. Jin, Y.; Duan, Y.L. A new method for abnormal underground rocks identification using ground penetrating radar. Measurement 2020, 149, 106988. [CrossRef]

7. Ibrahim, A.A.; Ata, S.O.; Erdogan, E.; Durak-Ata, L. Performance analysis of free space optical communication systems over imprecise Malaga fading channels. Opt. Commun. 2020, 457, 124694. [CrossRef]

8. Wang, Z.J.; Zhou, J.; Wang, J.Y. A novel Fault Diagnosis Method of Gearbox Based on Maximum Kurtosis Spectral Entropy Deconvolution. IEEE Access 2019, 7, 29520-29532. [CrossRef]

9. Wang, F.; Hu, G.J.; Du, T.; Sun, Y.D.; Hao, X.M.; Chen, J.R.; Wu, P. Performance research of mPPM-QPSK modulation signal for free space optical communication. Opt. Commun. 2020, 457, 124646. [CrossRef]

10. Zhang, Z.J.; Xie, H.; Tong, X.H.; Zhang, H.W.; Liu, Y.; Li, B.B. Denoising for satellite laser altimetry full-waveform data based on EMD-Hurst analysis. Int. J. Digit. Earth 2019. [CrossRef]

11. Wang, Z.J.; Zheng, L.K.; Du, W.H. A novel method for intelligent fault diagnosis of bearing based on capsule neural network. Complexity 2019. [CrossRef]

12. Wang, Z.J.; Du, W.H.; Wang, Z.Y. Research and Application of Improved Adaptive MOMEDA Fault Diagnosis Method. Measurement 2019, 140, 63-75. [CrossRef]

13. Wu, Q.Q.; Qiang, S.Z.; Li, X.C.; Wang, Y.Q. Lidar full-waveform decomposition based on the empirical mode decomposition and Gaussian function model. Meas. Sci. Technol. 2020, 31, 025206.

14. Cho, H.; Kim, W.J.; Hong, W. Underwater Signal Analysis in the Modulation Spectrogram with Time-Frequency Reassignment Technique. IEICE Trans. Fundam. Electron. Commun. Comput. Sci. 2019, E102A, 1542-1544. [CrossRef]

15. Auger, F.; Flandrin, P.; Lin, Y.T.; McLaughlin, S.; Meignen, S.; Oberlin, T.; Wu, H.T. Time-Frequency Reassignment and Synchrosqueezing. IEEE Signal Process. Mag. 2013, 30, 32-41. [CrossRef]

16. Li, L.; Cai, H.Y.; Han, H.X.; Jiang, Q.T.; Ji, H.B. Adaptive short-time Fourier transform and synchrosqueezing transform for non-stationary signal separation. Signal Process. 2020, 166, 107231. [CrossRef]

17. Tao, J.F.; Zeng, H.W.; Qin, C.J.; Liu, C.L. Chatter detection in robotic drilling operations combining multi-synchrosqueezing transform and energy entropy. Int. J. Adv. Manuf. Technol. 2019, 105, 2879-2890. [CrossRef]

18. Meignen, S.; Pham, D.H.; McLaughlin, S. On Demodulation, Ridge Detection, and Synchrosqueezing for Multicomponent Signals. IEEE Trans. Signal Process. 2017, 65, 2093-2103. [CrossRef]

19. Wang, S.B.; Chen, X.F.; Cai, G.G.; Chen, B.Q.; Li, X.; He, Z.J. Matching Demodulation Transform and SynchroSqueezing in Time-Frequency Analysis. IEEE Trans. Signal Process. 2014, 62, 69-84. [CrossRef] 
20. Tu, X.T.; Hu, Y.; Li, F.C.; Abbas, S.; Liu, Z.; Bao, W.J. Demodulated High-Order Synchrosqueezing Transform With Application to Machine Fault Diagnosis. IEEE Trans. Ind. Electron. 2019, 66, 3071-3081. [CrossRef]

21. Pham, D.H.; Meignen, S. High-Order Synchrosqueezing Transform for Multicomponent Signals Analysis-With an Application to Gravitational-Wave Signal. IEEE Trans. Signal Process. 2017, 65, 3168-3178. [CrossRef]

22. Daldal, N.; Comert, Z.; Polat, K. Automatic determination of digital modulation types with different noises using Convolutional Neural Network based on time-frequency information. Appl. Soft Comput. 2020, 86, 105834. [CrossRef]

23. Wang, Z.J.; Wang, J.Y.; Cai, W.N.; Zhou, J.; Du, W.H.; Wang, J.T.; He, G.F.; He, H.H. Application of an Improved Ensemble Local Mean Decomposition Method for Gearbox Composite Fault diagnosis. Complexity 2019. [CrossRef]

24. Wang, Z.J.; He, G.F.; Du, W.H.; Zhou, J.; Han, X.F.; Wang, J.T.; He, H.H.; Guo, X.M.; Wang, J.Y.; Kou, Y.F. Application of Parameter Optimized Variational Mode Decomposition Method in Fault Diagnosis of Gearbox. IEEE Access 2019, 7, 44871-44882. [CrossRef]

25. Prusa, Z.; Rajmic, P. Toward High-Quality Real-Time Signal Reconstruction From STFT Magnitude. IEEE Signal Process. Lett. 2017, 24, 892-896. [CrossRef]

26. Jevremovic, I.; Erbe, A. The reassigned pseudo Wigner-Ville transform in electrochemical noise analysis. Phys. Chem. Chem. Phys. 2019, 21, 24361-24372. [CrossRef]

27. Zoubi, A.B.; Kim, S.; Adams, D.O.; Mathews, V.J. Lamb Wave Mode Decomposition Based on Cross-Wigner-Ville Distribution and Its Application to Anomaly Imaging for Structural Health Monitoring. IEEE Trans. Ultrason. Ferroelectr. Freq. Control 2019, 66, 984-997. [CrossRef]

28. Wang, T.R.; Liu, N.; Su, Z.; Li, C. A New Time-Frequency Feature Extraction Method for Action Detection on Artificial Knee by Fractional Fourier Transform. Int. Trans. Electr. Energy Syst. 2019, 10, 333. [CrossRef]

29. Zayed, A. A New Perspective on the Two-Dimensional Fractional Fourier Transform and Its Relationship with the Wigner Distribution. J. Fourier Anal. Appl. 2019, 25, 460-487. [CrossRef]

30. Djurovic, I.; Simeunovic, M.; Wang, P. Cubic phase function: A simple solution to polynomial phase signal analysis. Signal Process. 2017, 135, 48-66. [CrossRef]

31. Justel, D. The Zak transform on strongly proper G-spaces and its applications. J. Lond. Math. Soc. 2018, 97, 47-76. [CrossRef]

32. Arun, K.R.; Yap, X.; Khong, A.W.H. A Touch Interface Exploiting Time-Frequency Classification Using Zak Transform for Source Localization on Solids. IEEE Trans. Multimed. 2011, 13, 487-497. [CrossRef]

33. Chen, S.; Dong, X.; Peng, Z. Nonlinear Chirp Mode Decomposition: A Variational Method. IEEE Trans. Signal Process. 2017, 65, 6024-6037. [CrossRef]

34. Chen, S.Q.; Yang, Y.; Peng, Z.K.; Wang, S.B.; Zhang, W.M.; Chen, X.F. Detection of rub-impact fault for rotor-stator systems: A novel method based on adaptive chirp mode decomposition. J. Sound Vib. 2019, 440, 83-99. [CrossRef]

35. Zhou, X.J.; Yang, C.H.; Gui, W.H. State transition algorithm. J. Ind. Ind. Manag. Optim. 2012, 8, $1039-1056$. [CrossRef]

36. Yin, J.T.; Xie, Y.F.; Chen, Z.W.; Peng, T.; Yang, C.H. Weak-fault diagnosis using state-transition-algorithm-based adaptive stochastic-resonance method. J. Cent. South Univ. 2019, 26, 1910-1920. [CrossRef]

37. Zhou, X.; Yang, C.; Gui, W. A Statistical Study on Parameter Selection of Operators in Continuous State Transition Algorithm. IEEE Trans. Cybern. 2019, 49, 3722-3730. [CrossRef]

38. Voices in the Sea. Available online: http://cetus.ucsd.edu/voicesinthesea_org/index.html (accessed on 21 November 2019).

(C) 2020 by the authors. Licensee MDPI, Basel, Switzerland. This article is an open access article distributed under the terms and conditions of the Creative Commons Attribution (CC BY) license (http://creativecommons.org/licenses/by/4.0/). 